\title{
Effects of neuroactive metabolites of the tryptophan pathway on working memory and cortical thickness in schizophrenia
}

\author{
Junchao Huang ${ }^{1}$, Jinghui Tong ${ }^{1}$, Ping Zhang ${ }^{1}$, Yanfang Zhou', Yimin Cui ${ }^{2}$, Shuping Tan' ${ }^{1}$, Zhiren Wang ${ }^{1}$, Fude Yang ${ }^{1}$,
} Peter Kochunov', Joshua Chiappelli3 , Baopeng Tian', Li Tian (1) ${ }^{4}$, Yunlong Tan (1) and L. Elliot Hong ${ }^{3}$

\begin{abstract}
A number of tryptophan metabolites known to be neuroactive have been examined for their potential associations with cognitive deficits in schizophrenia. Among these metabolites, kynurenic acid (KYNA), 5-hydroxyindole (5-HI), and quinolinic acid (QUIN) are documented in their diverse effects on a-7 nicotinic acetylcholine receptor (a7nAChR) and/ or $\mathrm{N}$-methyl-D-aspartate receptor (NMDAR), two of the receptor types thought to contribute to cognitive impairment in schizophrenia. In this study, serum levels of KYNA, 5-HI, and QUIN were measured in 195 patients with schizophrenia and in 70 healthy controls using liquid chromatography-tandem mass spectrometry; cognitive performance in MATRICS Consensus Cognitive Battery and cortical thickness measured by magnetic resonance imaging were obtained. Patients with schizophrenia had significantly lower serum KYNA $(p<0.001)$ and QUIN $(p=0.02)$ levels, and increased 5-HI/KYNA $(p<0.001)$ and QUIN/KYNA ratios $(p<0.001)$ compared with healthy controls. Multiple linear regression showed that working memory was positively correlated with serum 5 -HI levels $(t=2.10, p=0.04)$, but inversely correlated with KYNA concentrations $(t=-2.01, p=0.05)$ in patients. Patients with high 5 -HI and low KYNA had better working memory than other subgroups $(p=0.01)$. Higher 5 -HI levels were associated with thicker left lateral orbitofrontal cortex $\left(t=3.71, p=2.94 \times 10^{-4}\right)$ in patients. The different effects of 5 -HI and KYNA on working memory may appear consistent with their opposite receptor level mechanisms. Our findings appear to provide a new insight into the dynamic roles of tryptophan pathway metabolites on cognition, which may benefit novel therapeutic development that targets cognitive impairment in schizophrenia.
\end{abstract}

\section{Introduction}

The tryptophan pathway has been increasingly targeted in drug discovery efforts for treating cognitive impairments in neuropsychiatric conditions including schizophrenia ${ }^{1,2}$. One of the metabolites in this pathway is kynurenic acid (KYNA), which has attracted considerable interest, as it is a noncompetitive antagonist of $\alpha-7$ nicotinic acetylcholine receptor $(\alpha 7 \mathrm{nAChR})^{3,4}$ that has been linked to cognitive functions, in particular working memory and attention ${ }^{5,6}$.

\footnotetext{
Correspondence: Yunlong Tan (yltan21@126.com)

${ }^{1}$ Peking University HuiLongGuan Clinical Medical School, Beijing Huilongguan Hospital, Beijing, China

${ }^{2}$ Department of Pharmacy, Peking University First Hospital, Beijing, China

Full list of author information is available at the end of the article
}

Accumulated evidences suggest that schizophrenia is associated with abnormal $\alpha 7 \mathrm{nAChR}$-mediated neurotransmis$\operatorname{sion}^{7,8}$ and cortical KYNA contributes to cognitive impairment through the $\alpha 7 \mathrm{nAChR}$ mechanism ${ }^{3,9-11}$. KYNA also antagonizes the glycine-binding site of the $N$-methyl-Daspartate receptor (NMDAR) $)^{4,12}$ and NMDAR dysfunction has been linked to cognition, especially working memory deficits in schizophrenia ${ }^{13-16}$.

Interestingly, another neuroactive metabolite in the tryptophan pathway, 5-hydroxyindole (5-HI), also affects $\alpha 7 \mathrm{nAChR}^{17-19} .5-\mathrm{HI}$ is a lipophilic solute and was shown to permeate epithelial plasma membrane ${ }^{20}$, implying that peripheral 5 -HI may penetrate the blood-brain barrier (BBB). In contrast to KYNA, 5 -HI potentiates $\alpha 7 \mathrm{nAChR}$ -

\section{(c) The Author(s) 2021}

\footnotetext{
(c) Open Access This article is licensed under a Creative Commons Attribution 4.0 International License, which permits use, sharing, adaptation, distribution and reproduction cc) in any medium or format, as long as you give appropriate credit to the original author(s) and the source, provide a link to the Creative Commons license, and indicate if changes were made. The images or other third party material in this article are included in the article's Creative Commons license, unless indicated otherwise in a credit line to the material. If material is not included in the article's Creative Commons license and your intended use is not permitted by statutory regulation or exceeds the permitted use, you will need to obtain permission directly from the copyright holder. To view a copy of this license, visit http://creativecommons.org/licenses/by/4.0/.
} 


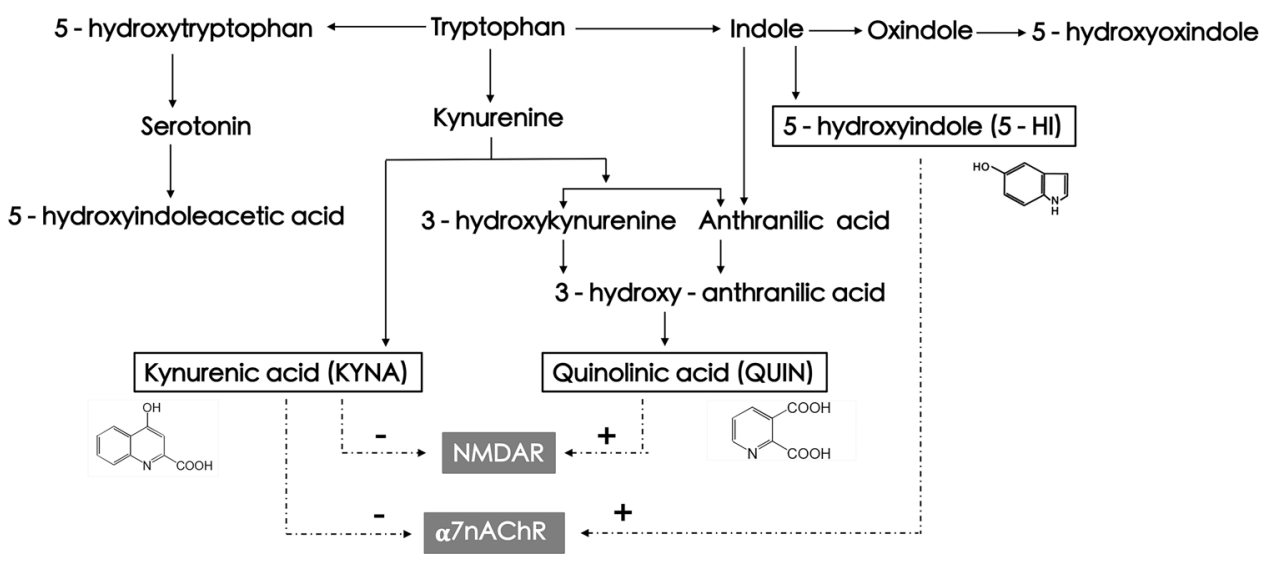

Fig. 1 Abbreviated tryptophan pathway and the presumed neuroactive metabolite effects on a7nAChR and NMDAR. Dotted line refers to hypothetical effects based on preclinical data. Positive signs show the agonist effect and negative signs show the antagonist effect ${ }^{17,49,89,90}$.

mediated electrophysiological responses and $\mathrm{Ca}^{2+}$ influx in a concentration-dependent manner ${ }^{18,19,21}$. Furthermore, 5-HI-potentiated $\alpha 7 \mathrm{nAChR}$ activations are dosedependently downregulated by KYNA ${ }^{19}$. Hence, these two metabolites of the tryptophan pathway counteract on $\alpha 7 \mathrm{nAChR}$-mediated functions and may form an opposing effect on working memory and other cognitive functions mediated by $\alpha 7 \mathrm{nAChR}$ (Fig. 1).

5-HI and KYNA may also diverge on their roles in cognition, as they may modulate $\gamma$-aminobutyric acid (GABA)ergic neurotransmission differently, as 5-HI facilitates GABAergic transmission via excitation of $\alpha 7 \mathrm{nAChR}^{22}$, but KYNA concentration-dependently reduces GABA levels by modulating $\alpha 7 \mathrm{nAChR}$ function $^{23}$. GABAergic dysfunction has also been consistently linked to working memory deficits in schizophrenia ${ }^{24-26}$.

Notably, a third metabolite in the tryptophan pathway, quinolinic acid (QUIN), is an agonist of $\mathrm{NMDAR}^{27,28}$. QUIN is an excitotoxin that promotes neurodegeneration and neuroinflammation, and may negatively impact cognition $^{29-31}$.

Therefore, 5-HI, KYNA, and QUIN are three neuroactive metabolites within the tryptophan pathway, which may potentially influence cognition, although their in vivo combined effects are likely complex and are currently unknown. The effort of targeting the tryptophan pathway for treating cognitive deficits in schizophrenia should first understand the independent vs. joint effects of these neuroactive metabolites. Accordingly, we tested the hypothesis that working memory in schizophrenia may be dynamically dependent on the balance among 5-HI, KYNA, and QUIN. As these metabolites are known to influence $\alpha 7 \mathrm{nAChR}$ and $\mathrm{NMDAR}^{32,33}$, which are distributed in high densities in the cortex $^{34,35}$, we will test a second hypothesis that they may also counteractively impact the cortical thickness in patients with schizophrenia.

\section{Methods \\ Participants}

The study recruited 195 patients with schizophrenia (all in-patients) and 70 healthy controls ( $\mathrm{HCs}$ ). Patients who met the diagnostic criteria of schizophrenia according to the Structured Clinical Interview of the Diagnostic and Statistical Manual of Mental Disorders-IV were enrolled during their hospitalization at the Beijing Huilongguan Hospital. HCs were recruited through local advertisements at nearby communities and were excluded if they had a history of psychiatric disorders or psychosis among their first-degree relatives. Participants were also excluded if they had a lifetime history of any other Axis I disorders, head trauma, current or previous substance or alcoholism dependence (except nicotine), or systemic diseases including neurological disorders, organic brain disorders, and unstable medical illnesses. One hundred and twelve patients were antipsychotic medication-free at the time of admission and blood sample collection; 19 patients were on a first-generation antipsychotic alone or combined with second-generation antipsychotics; and the remaining patients were on the following second-generation antipsychotics: risperidone (14), clozapine (5), olanzapine (5), and aripiprazole (4). Thirty-six patients were on two or more second-generation antipsychotics and four patients were also on sodium valproate. Patients received antipsychotic medications once hospitalized and, most initially, medication-free patients were on antipsychotic medications during brain imaging, which was on average $5.5 \pm 2.3$ days after the initial blood draw. The current antipsychotic medication dose (based on time of blood draw) was calculated as a chlorpromazine (CPZ)-equivalent dose $^{36,37}$ (Table 1). This study was approved by the ethics committee and institutional review board of Beijing Huilongguan Hospital. All participants provided written informed consent. 
Table 1 Participant demographics, clinical characteristics, and metabolic measures.

\begin{tabular}{|c|c|c|c|c|}
\hline & Schizophrenia $(n=195)$ & Healthy controls $(n=70)$ & Test statistic & $p$-Value \\
\hline Male/female & $110 / 85$ & $37 / 33$ & $x^{2}=0.26$ & 0.61 \\
\hline Smoker/non-smoker & $52 / 143$ & $17 / 53$ & $x^{2}=0.15$ & 0.70 \\
\hline Age (years) & $35.60(13.03)$ & $39.74(11.82)$ & $t=2.33$ & 0.02 \\
\hline Education (years) & $12.37(3.21)$ & $12.91(2.56)$ & $t=1.28$ & 0.20 \\
\hline BMI & $23.43(4.36)$ & $23.92(3.07)$ & $t=1.02$ & 0.31 \\
\hline $\mathrm{CPZ}$ & $266.31(349.77)$ & NA & NA & NA \\
\hline PANSS total score & $72.36(17.13)$ & NA & NA & NA \\
\hline Working memory ${ }^{a}$ & $45.16(11.43)$ & $57.78(7.65)$ & $F=78.19$ & $<0.001$ \\
\hline Processing speed ${ }^{\mathrm{a}}$ & $44.96(8.73)$ & $57.09(8.51)$ & $F=92.99$ & $<0.001$ \\
\hline Attention/vigilance ${ }^{a}$ & $43.64(9.85)$ & $56.81(9.08)$ & $F=59.66$ & $<0.001$ \\
\hline$V_{\text {Verbal learning }}{ }^{\mathrm{a}}$ & $46.98(12.17)$ & $57.41(8.34)$ & $F=39.31$ & $<0.001$ \\
\hline$V_{\text {Visual learning }}{ }^{a}$ & $45.23(10.56)$ & $53.39(8.47)$ & $F=37.73$ & $<0.001$ \\
\hline Reasoning and problem solving ${ }^{a}$ & $45.70(10.70)$ & $55.88(7.81)$ & $F=69.88$ & $<0.001$ \\
\hline Social cognition ${ }^{a}$ & $46.20(10.97)$ & $53.22(9.95)$ & $F=24.76$ & $<0.001$ \\
\hline MCCB composite score ${ }^{a}$ & $43.91(10.21)$ & $57.83(7.93)$ & $F=103.64$ & $<0.001$ \\
\hline $5-\mathrm{Hl}(\mathrm{ng} / \mathrm{ml})^{\mathrm{a}}$ & $8.57,8.44(2.79)$ & $7.85,7.73(2.12)$ & $F=2.54$ & 0.11 \\
\hline KYNA $(n g / m l)^{a}$ & $6.42,5.77(3.36)$ & $8.59,8.14(3.09)$ & $F=34.38$ & $<0.001$ \\
\hline QUIN (ng/ml) ${ }^{\mathrm{a}}$ & $49.62,44.71(24.73)$ & $54.18,50.51(16.55)$ & $F=5.63$ & 0.02 \\
\hline $5-H I / K Y N A^{a}$ & $1.69,1.36(1.30)$ & $1.01,0.94(0.40)$ & $F=33.61$ & $<0.001$ \\
\hline QUIN/KYNA ${ }^{a}$ & $8.67,8.04(3.8)$ & $6.77,6.36(2.44)$ & $F=18.05$ & $<0.001$ \\
\hline
\end{tabular}

Data reported as [mean (SD)], except for 5-HI, KYNA, QUIN, 5-HI/KYNA, and KYNA/QUIN as [mean, median (SD)].

$C P Z$ chlorpromazine equivalent, DBP diastolic blood pressure, 5-HI 5-hydroxyindole, KYNA kynurenic acid, MCCB MATRICS Consensus Cognitive Battery, NA not applicable, PANSS Positive and Negative Syndrome Scale, QUIN quinolinic acid, SBP systolic blood pressure.

${ }^{a}$ Statistics included sex and age as covariates.

\section{Clinical and cognitive assessments}

Clinical symptoms were evaluated by the Positive and Negative Syndrome Scale (PANSS) in patients of schizophrenia by one of the three attending psychiatrists. The inter-rater intra-class correlation coefficient among the raters was above 0.80. Cognitive function was assessed using the validated Chinese version of the MATRICS Consensus Cognitive Battery $(\mathrm{MCCB})^{38-40}$. The MCCB contains assessments of seven cognitive domains: Working Memory, Speed of Processing, Attention and Vigilance, Verbal Learning, Visual Learning, Reasoning and Problem Solving, and Social Cognition. Initial scores were converted to domain scores. Impaired working memory was evidenced a pervasive and the core of schizophreniarelated cognitive disability ${ }^{41,42}$; thus, our primary focus was on the working memory domain.

\section{Biochemistry}

Blood samples were collected with BD Vacutainer serum tubes in the morning after overnight fasting and centrifuged immediately at $4{ }^{\circ} \mathrm{C}$ for $10 \mathrm{~min}$ at 3000 r.p.m.
Serum was then aliquoted into separate tubes and stored at $-80{ }^{\circ} \mathrm{C}$. High-performance liquid chromatography was used to separate serum KYNA, 5-HI, and QUIN, and tandem mass spectrometry was then performed to quantify them using standard protocols. Details of the 5HI assay, quality control, and validations were in Supplementary Material 1. Details of the KYNA and QUIN assays were described as previous reports ${ }^{43-45}$. The intratrial coefficient of variations for high, median, and low quality were $1.2 \%, 2.7 \%$, and $1.5 \%$ for $5-\mathrm{HI} ; 4.0 \%, 12.5 \%$, and $8.8 \%$ for KYNA; and $4.1 \%, 6.2 \%$, and $7.4 \%$ for QUIN, respectively. We further calculated the 5-HI/KYNA ratio and the QUIN/KYNA ratio to explore their putative agonistic/antagonistic effects on $\alpha 7 \mathrm{nAChR}$ and NMDAR, respectively.

\section{Image processing}

Structural images were collected in 153 schizophrenia patients and 65 HCs using a 3.0 Tesla Prisma MRI scanner (Siemens, Germany) and a 64-channel head coil at the MRI Research Center of the Beijing Huilongguan 
Hospital. Parameters of the three-dimensional magnetization prepared rapid acquisition gradient echo sequence were as follows: echo time $=2.98 \mathrm{~ms}$, inversion time $=$ $1100 \mathrm{~ms}$, repetition time $=2530 \mathrm{~ms}$, flip angle $=7^{\circ}$, field of view $=256 \times 224 \mathrm{~mm}^{2}$, matrix size $=256 \mathrm{~mm} \times$ $224 \mathrm{~mm}$, and thickness/gap $=1 / 0 \mathrm{~mm}$ covering the whole brain. Participants used earplugs and foam pads to reduce magnetic machine noise and head movement, and who were reminded not to move their head during the procedure. A computer-connected monitor was acquired to detect head movement distance. The automated and validated segmentation were conducted by using FreeSurfer v5.3 (http://surfer.nmr.mgh.harvard.edu/ $/)^{46}$. Thirty-four cortical gray matter regions in each hemisphere were extracted as regions of interest for statistical analysis according to the Desikan-Killiany atlas ${ }^{47,48}$.

\section{Statistical analysis}

Normality of raw data was assessed with normal $Q-Q$ plots and Shapiro-Wilkinson tests. Serum 5-HI, KYNA, QUIN levels, and 5-HI/KYNA ratio all deviated from normal distribution; all were normalized after natural logarithm (ln) or square root (sqrt) transformation. $T$ tests or $\chi^{2}$-test were used to compare the demographic data of patients with schizophrenia and HCs. Separate univariate analyses of covariance (ANCOVAs) were used to determine between-group differences in metabolites, MCCB and PANSS subdomain, and total scores, with sex and age as covariates. Multiple linear regression model was used to test whether working memory score was associated with the serum levels of 5-HI, KYNA, and QUIN in schizophrenia patients, adjusted for age and sex. Significant but opposite directions of associations of the metabolites with working memory would imply opposite roles. For those metabolites having significant opposite effects on working memory, we further divided patients into high- (concentration equal to or above the median) and low- (concentration below the median) level subgroups, and compared their working memory performance, adjusted for age and sex. Associations between cortical thickness of 68 cortical regions and serum levels of 5-HI, KYNA, and QUIN were further explored by multiple linear regression adjusted by age and sex in patients. These analyses were also repeated in HCs. The effects of smoking status and psychotropic medications on the three metabolites were also explored. Significance was set at $p<0.05$ in all tests.

\section{Results}

\section{Group differences}

The summary demographics, clinical characteristics, and outcome variables were presented in Table 1. After controlling for sex and age, all seven domains and $\mathrm{MCCB}$ total score were lower in patients with schizophrenia compared to HCs $(F=24.76-78.19, p<0.001)$. Patients with schizophrenia also had significantly lower serum KYNA $(F=34.38, p<0.001)$ and QUIN $(F=5.63, p=$ $0.02)$ than in $\mathrm{HCs}$, but 5 -HI concentrations did not differ between groups $(F=2.54, p=0.11)$. 5-HI/KYNA $(F=$ 33.61, $p<0.001)$ and QUIN/KYNA $(F=18.05, p<0.001)$ were significantly higher in patients with schizophrenia than in HCs. Data were also analyzed in medication-free patients. Medication-free patients showed significantly lower KYNA $(5.36 \pm 2.50 \mathrm{ng} / \mathrm{mL}$ vs. $7.31 \pm 3.84 \mathrm{ng} / \mathrm{mL} ; F$ $=11.87, p=0.001)$ and QUIN $(41.60 \pm 12.26 \mathrm{ng} / \mathrm{mL}$ vs. $57.67 \pm 30.89 \mathrm{ng} / \mathrm{mL} ; F=8.09, p=0.01)$, but insignificant on 5 - HI $(8.63 \pm 2.95 \mathrm{ng} / \mathrm{mL}$ vs. $8.44 \pm 2.77 \mathrm{ng} / \mathrm{mL} ; F=$ $0.35, p=0.55)$ levels compared to patients on antipsychotic medications. As medication-free patients have even lower KYNA and QUIN levels than patients on antipsychotic medications, the low serum levels of KYNA and QUIN in schizophrenia are unlikely due to antipsychotic medication effects.

\section{Relationship of working memory with $5-\mathrm{HI}$, KYNA, and QUIN}

The multiple linear regression model using the three metabolites as predictors was significant $(F=3.18, p=$ 0.01 ), wherein working memory was significantly predicted by higher level of 5 - $\mathrm{HI}(t=2.10, p=0.04)$ and lower KYNA $(t=-2.01, p=0.05)$ (Table 2). The results were not statistically significant, although 5 - $\mathrm{HI}(t=0.94$, $p=0.35)$ and KYNA $(t=-1.48, p=0.14)$ had the same trend in the medication-free group, similar to findings in the total-patient group. One possible reason is that each divided group may have lower statistic power than the total-patient group. The results were still significant $(F=$ 10.11, $p<0.001$ ) when we added smoke and CPZ equivalent in the model, and 5 - $\mathrm{HI}(t=2.18, p=0.03)$ and KYNA $(t=-2.24, p=0.03)$ have the same opposite trends as before. The model was insignificant in HCs

Table 2 Multiple regression analysis results of serum levels of 5-HI, KYNA, and QUIN on working memory score.

\begin{tabular}{|c|c|c|c|c|c|c|}
\hline & \multicolumn{3}{|l|}{ Schizophrenia } & \multicolumn{3}{|l|}{ Healthy controls } \\
\hline & Standardized $\beta$ & $t$ & $p$ & Standardized $\beta$ & $t$ & $P$ \\
\hline $5-\mathrm{HI}$ & 0.15 & 2.10 & 0.04 & -0.02 & -0.19 & 0.85 \\
\hline KYNA & -0.18 & -2.01 & 0.05 & -0.02 & -0.14 & 0.89 \\
\hline QUIN & 0.06 & 0.68 & 0.49 & 0.11 & 0.75 & 0.46 \\
\hline Sex & 0.16 & 2.04 & 0.04 & 0.16 & 1.18 & 0.24 \\
\hline Age & -0.02 & -0.26 & 0.80 & -0.10 & -0.10 & 0.92 \\
\hline Model & \multicolumn{3}{|l|}{$F=3.18, p=0.01$} & \multicolumn{3}{|l|}{$F=0.42, p=0.83$} \\
\hline
\end{tabular}

5-HI 5-hydroxyindole, KYNA kynurenic acid, QUIN uinolinic acid. Bold values indicates statistical significance at $p<0.05$. 

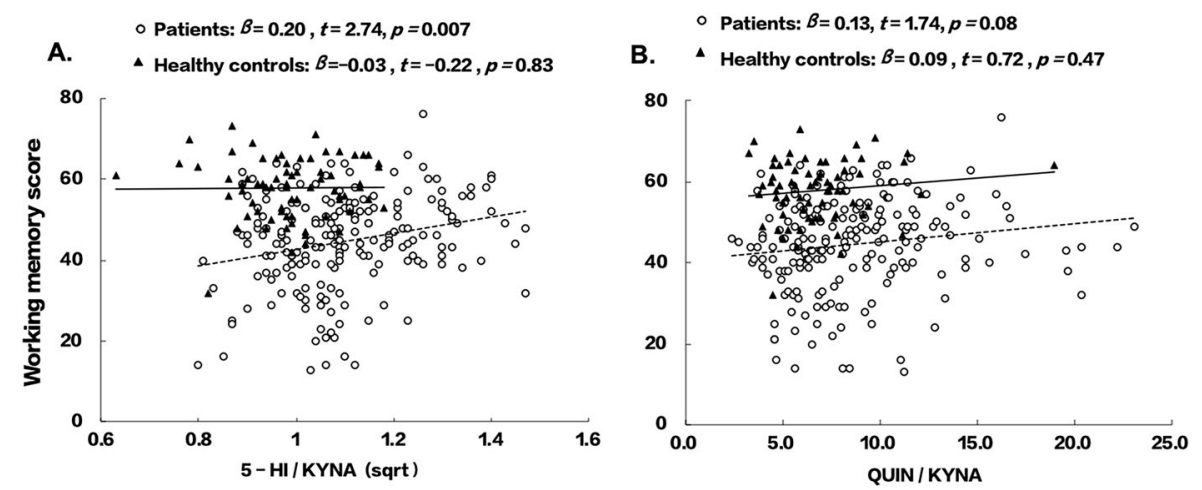

Fig. 2 Relationship between working memory performance and 5-HI/KYNA or QUIN/KYNA. A Relationship of working memory with 5-HI/ KYNA. B Relationship of working memory with QUIN/KYNA.

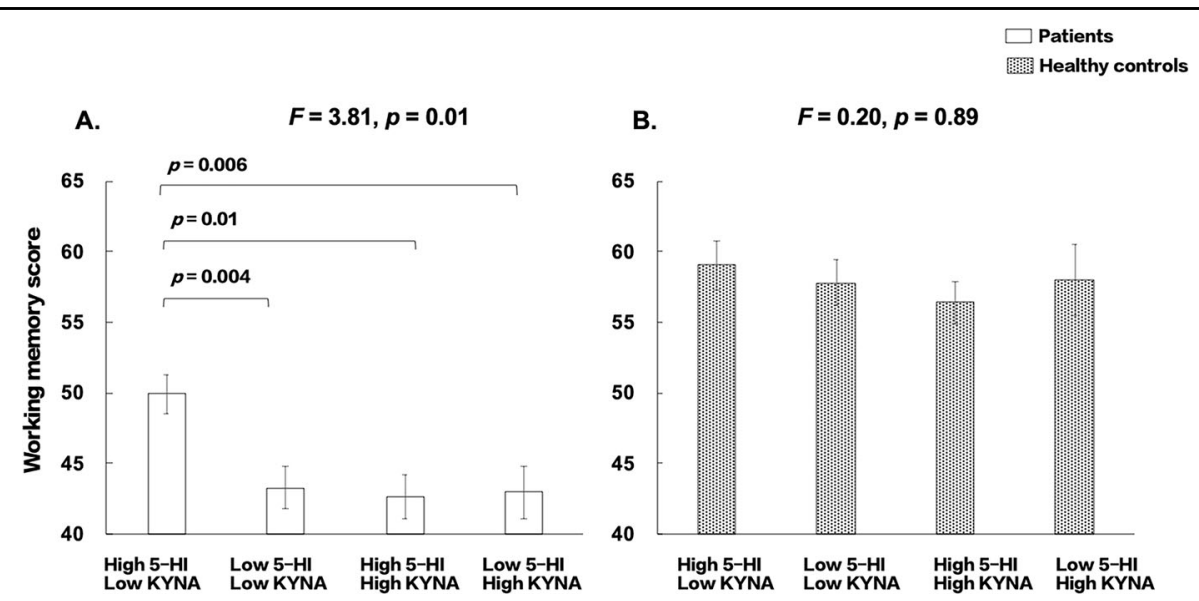

Fig. 3 Comparisons of working memory performance in subgroups with 5-HI and KYNA levels by medium splits adjusting for age and sex. A Patients with schizophrenia. B Healthy controls.

(model $p=0.83$ ). We also explored other MCCB domains and total score but found no significant results in either patients or controls (Supplementary Table 2).

The relationships of working memory with $5-\mathrm{HI} / \mathrm{KYNA}$ and QUIN/KYNA were also explored. 5-HI/KYNA was positively associated with working memory $(t=2.74, p=$ 0.007 ) in patients, but not in HCs (Fig. 2). There was no significant correlation with QUIN/KYNA in either patients or controls.

We further divided the patients into high- and low-level in four subgroups based on medium splits for 5-HI and KYNA: high 5-HI/low KYNA, low 5-HI/low KYNA, high 5-HI/high KYNA, and high 5-HI/low KYNA (Fig. 3). The overall ANCOVA was significant $(F=3.81, p=0.01)$. Post-hoc tests showed that patients with high $5-\mathrm{HI}$ and low KYNA had the best working memory performance compared with the other three subgroups $(p=$ 0.01-0.004); however, the other three subgroups did not significantly differ. The model was not significant in $\mathrm{HCs}$ $(F=0.20, p=0.89)$.

\section{Relationship of cortical thickness with 5-HI, KYNA, and QUIN}

Multiple cortical regions showed significantly reduced cortical thickness in patients compared to HCs (Supplementary Table 3). We next explored the associations of 5HI, KYNA, and QUIN with thickness of 68 cortical regions after controlling for sex and age. Only the left lateral orbitofrontal cortex (LOFC) showed significantly positive association with the 5 -HI levels $(t=3.71, p=$ $2.94 \times 10^{-4}$ ) after Bonferroni correction for multiple comparisons $\left(0.05 / 68=7.35 \times 10^{-4}\right)$ (Fig. 4). However, we also observed nominally significant $(p<0.05$ uncorrected) positive associations between $5-\mathrm{HI}$ and the left and right frontal poles, left insula, right caudal-middle frontal, right lateral orbitofrontal, and right lingual areas in patients with schizophrenia $(p=0.03-0.004)$, whereas the right lateral occipital region had a nominally negative association with 5-HI $(p=0.05)$ (Supplementary Table 4$)$. In medication-free patients, 5 -HI was still correlated with LOFC thickness $\left(r=0.39, p=1.4 \times 10^{-4}\right)$. There were no 


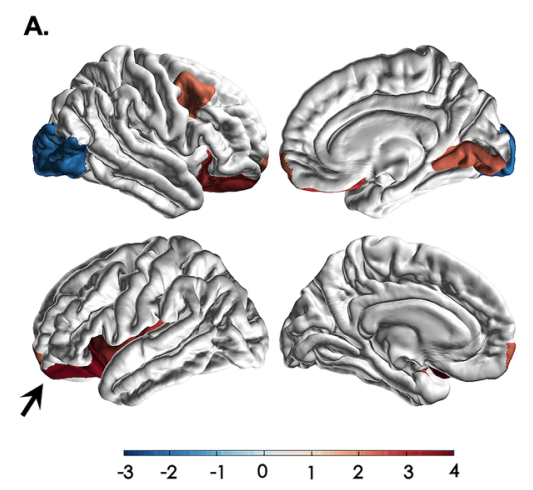

B. $\quad B=0.24, t=3.71, p=2.94 \times 10^{-4}$

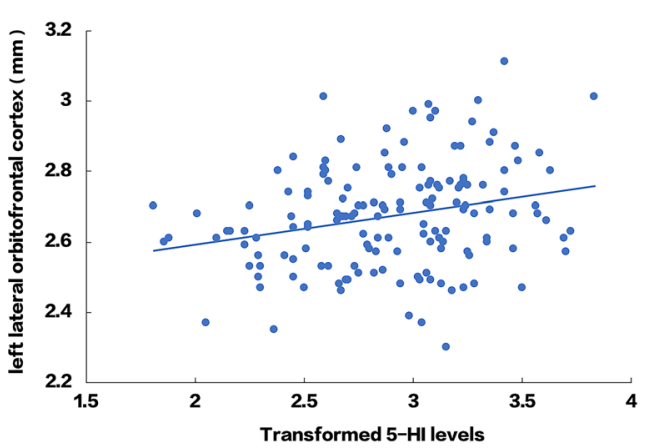

Fig. 4 The relationship of serum levels of 5-HI with cortical thickness in patients with schizophrenia. The left and right frontal poles, left insula, right caudal-middle frontal, left and right lateral orbitofrontal, and right lingual areas were positively and significantly associated with 5 -HI at $p=$ 0.03-0.0003, and the right lateral occipital region was inversely associated with $5-\mathrm{HI}$ at $p=0.05$, but only the lateral orbitofrontal cortex (arrow) was significant after correction for multiple comparisons. A Cortical regions were colored according to $t$-scores by multiple linear regression. The color bar represents $t$-scores. B Scatter plot between $5-\mathrm{HI}$ and the left lateral orbitofrontal cortex (arrow in $\mathbf{A}$ ) in patients with schizophrenia.

significant associations between cortical thickness and 5$\mathrm{HI}$ in HCs. No significant associations between cortical thickness and KYNA, QUIN, or their ratios were found in patients or controls after correction for multiple comparisons (Supplementary Table 4).

The left LOFC thickness was positively associated with working memory $(t=2.68, p=0.008)$. The left LOFC thickness was also associated with verbal learning ( $t=$ 4.27, $p<0.001)$, social cognition $(t=3.05, p=0.003)$, and reasoning and problem solving ( $t=2.47, p=0.02$ ) (Supplementary Table 5).

\section{Relationship of tryptophan metabolites with sex, age, smoking, blood pressure, heart rate, and medication}

After controlling for sex and age, CPZ was negatively correlated with 5-HI $(r=-0.24, p=0.04)$, but not with KYNA or QUIN (both $p>0.05$ ). Males had higher 5-HI $(8.70 \pm 2.58 \mathrm{ng} / \mathrm{mL})$, KYNA $(7.65 \pm 3.65 \mathrm{ng} / \mathrm{mL})$, and QUIN $(54.34 \pm 26.23 \mathrm{ng} / \mathrm{mL})$ concentrations than females $(7.93 \pm 2.75,5.86 \pm 2.66$, and $45.45 \pm 15.29 \mathrm{ng} /$ $\mathrm{mL}$, respectively) $(F=6.19, p=0.01 ; F=25.68, p<$ $0.001 ; F=10.40, p=0.001$, respectively), but $\operatorname{sex} \times$ diagnosis interactions were insignificant (all $p>0.20$ ). Non-smokers had significantly lower KYNA concentrations than smokers $(6.57 \pm 3.27 \mathrm{ng} / \mathrm{mL}$ vs. $7.55 \pm$ $3.45 \mathrm{ng} / \mathrm{mL} ; F=6.67, p=0.01)$, but smoking $\times$ diagnosis interaction was insignificant $(p=0.36)$. Smokers and non-smokers did not significantly differ in 5-HI ( $p$ $=0.66)$ or QUIN $(p=0.31)$ levels, nor were the interactions of smoking with diagnosis $(p=0.37$ and 0.15 , respectively). Age was significantly correlated with KYNA $(r=0.13, p=0.03)$ and QUIN $(r=0.18, p=$ $0.001)$ concentrations, but not with 5 - HI $(p=0.06)$. We examined systolic and diastolic blood pressure, and heart rate in relation to KYNA, 5-HI, and QUIN, and found that there were insignificant correlations in either patients or HCs (all $p$ 's >0.05).

\section{Discussion}

This study examined the effects of 5-HI, KYNA, and QUIN on working memory and cortical structures, based on preclinical evidence of their effects on $\alpha 7 \mathrm{nAChR}$ and NMDAR. In patients with schizophrenia, KYNA and QUIN, but not 5-HI, were lower than in HCs. Working memory was significantly influenced by $5-\mathrm{HI}$ and KYNA but in opposite direction in the patients. Patients exhibiting high 5-HI and low KYNA concentrations had better working memory performance than the other subgroups. Finally, 5-HI level was positively associated with cortical thickness of the left orbitofrontal cortex, which was significantly associated with working memory performance in the patients.

5 - $\mathrm{HI}$ is a relatively potent tryptophan metabolite that increases glutamate release and the function of GABA interneurons $^{21}$, and in high dose can cause convulsion ${ }^{49}$. 5 -HI precursor indole is transformed from tryptophan by tryptophanase in indole-producing bacteria of human gut $^{50,51}$. Diverse oxygenase could degrade indole to indole derivatives $^{52,53}$ such as 5 - $\mathrm{HI}$. The formation of 5-HI can be observed by incubating rat liver homogenates with its direct precursor indole, supporting an endogenous production of $5-\mathrm{HI}^{21}$. In rats with hepatic encephalopathy, indole produced by gut bacteria is absorbed and metabolized into several metabolites including $5-\mathrm{HI}$, which may accumulate in the blood and brain ${ }^{17,21,54}$. Earlier clinical research has suggested that blood 5-HI may provide treatment effect monitoring for hyperactive behaviors, provided some initial support for its potential clinical utility ${ }^{55}$. Previous rodent studies indicated that 5$\mathrm{HI}$ activates both presynaptic and postsynaptic $\alpha 7 \mathrm{nAChR}$ 
to mediate glutamate release ${ }^{18,19,22}$, which may facilitate working memory. In addition, 5-HI increases acetylcholine (ACh) efficacy though $\mathrm{Ca}^{2+}$ currents, indicating that 5 - $\mathrm{HI}$ and ACh may cooperate to influence $\alpha 7 \mathrm{nAChR}{ }^{18}$. Regardless of whether our finding here is related to $\alpha 7 \mathrm{nAChR}$, the data provided the first evidence in humans, supporting the hypothesis that 5-HI may facilitate working memory performance in patients with schizophrenia.

Reduced serum KYNA concentration in schizophrenia was highly replicable in several recent reports ${ }^{56-58}$, which were not consistent with several earlier studies ${ }^{59,60}$. Our results are also inconsistent with studies showing elevated KYNA in the post-mortem brains of patients with schizophrenia $^{4,45,61}$. As KYNA normally does not pass the $\mathrm{BBB}^{62}$, it is unclear whether the observed inverse correlation between serum KYNA levels and working memory is due to these brain mechanisms; as such, the KYNArelated results should be viewed with caution. However, impaired BBB has been proposed in patients with schizophrenia $^{63,64}$, which may explain this significant correlation only in patients but not in controls. Moreover, evidences showed peripheral kynurenine can pass through $\mathrm{BBB}^{62,65}$ and may influence brain functions ${ }^{2,66}$. Preclinical studies have demonstrated that KYNA negatively affects $\alpha 7 n A C h R$-dependent presynaptic mechanisms in the prefrontal cortex and disrupts local GABAergic synaptic signaling ${ }^{67}$. Knocking out kynurenine aminotransferase II that decreases endogenous brain KYNA can increase working memory performance ${ }^{68,69}$. At high concentrations, KYNA is a competitive antagonist of $\mathrm{NMDAR}^{70,71}$; however, at low concentrations, KYNA has a more potent inhibitory effect on $\alpha 7 \mathrm{nAChR}$ activation than on $\mathrm{NMDAR}^{4}$. Furthermore, reducing KYNA increases 5-HIdependent activation of $\alpha 7 \mathrm{nAChR}{ }^{19}$. Therefore, our data appear as corroborating these preclinical data on the opposing 5 -HI vs. KYNA effects, showing that patients having high 5-HI and low KYNA was associated with the best working memory.

However, why patients have reduced serum KYNA but there is an inverse correlation between KYNA and working memory is difficult to interpret. Similar to our finding, significantly low peripheral serum kynurenine and/or KYNA levels have been found in diverse cohorts of patients $^{56-58,72}$. Meanwhile, higher KYNA in the brain is thought to impair cognitive functions ${ }^{2}$, which appears consistent with our finding of an inverse correlation between peripheral KYNA and working memory. We believe that the difficulty to explain the seemingly contradictory findings is in part due to the lack of a good explanation on why peripheral kynurenine and/or KYNA are reduced in schizophrenia, while brain KYNA appears increased in schizophrenia, compared with that in HCs. Further complicating the issue is that about $80 \%$ of kynurenine and KYNA in the blood were bound to albumin or other circulating binding proteins ${ }^{62,73}$, which may lead to differential availability of free kynurenine and KYNA, and our study is limited by not measuring specifically the free serum kynurenine or KYNA. Basic neuroscience effort to simultaneously assessing central and peripheral kynurenine and KYNA, while invasively studying the potential mechanism underlying the central-peripheral metabolite relationships may be needed.

We observed that serum QUIN concentration was significantly lower in patients with schizophrenia than in HCs. Previous studies attempted to identify QUIN-related abnormalities in schizophrenia but largely failed to show a significant differences in QUIN levels between patients and controls in post-mortem brain tissues ${ }^{45}$, blood $^{57}$, or $\mathrm{CSF}^{74}$. However, QUIN normally also does not pass the $\mathrm{BBB}^{62}$ and our analysis did not show significant relationship between QUIN and working memory or other clinical and cognitive measures.

An association between lower 5-HI to thinner cortical thickness of the LOFC in patients is intriguing. Previous researches indicated that the orbitofrontal cortex supports working memory ${ }^{75}$, specifically encoding gustatory ${ }^{75}$, emotional $^{76}$, and abstract information ${ }^{77}$. The LOFC is particularly important for reward learning ${ }^{78}$, a process closely related to working memory ${ }^{79}$. We consider the finding linking 5-HI to the thickness of the LOFC a further supportive evidence of a potential cognition enhancement effect of this metabolite in patients. Also some evidences showed relationships between kynurenine and subcortical volumes in mental disorders that KYNA/ 3-hydroxykynurenine were positively correlated to the hippocampal volume in bipolar disorder ${ }^{80}$ and negatively correlated with the left hippocampal activity in major depressive disorder ${ }^{81}$. We further explored the associations between subcortical volumes, and KYNA and KYNA/QUIN. However, there was no significant results in patients or in HCs (Supplementary Material 6).

The study has a number of limitations. We did not test whether the effects of these metabolites occurred through $\alpha 7 \mathrm{nAChR}, \mathrm{NMDAR}$, or other receptor mechanisms in the brain, although $5-\mathrm{HI}$ is lipophilic and may be BBB permeable ${ }^{20}$. However, KYNA and QUIN do not normally pass the BBB, making the blood-based finding or the lack thereof difficult to explain. We also did not measure the 5-HI precursor indole (Fig. 1) to rule out its contributions to the observations here. However, indole itself was thought not to interact with GABAergic or ionotropic glutamate receptors ${ }^{17}$. Furthermore, we measured 5hydroxyoxindole $(5-\mathrm{HOI})$, another metabolite from the indole and oxindole branch (Fig. 1), to confirm that the 5HI results reported here is distinct from 5-HOI (Supplementary Material 7). We also did not measure the gut flora that possibly influence the concentration of indole 
and further related to 5-HI level. The inflammatory markers were not tested in the present study, which may limit the interpretation of relationship between metabolites, as high inflammatory activity can lead to elevations of kynurenine and KYNA ${ }^{82-84}$. We also did not measure diet and physical activities that may have an effect on peripheral levels of KYNA ${ }^{85,86}$, cortical thickness ${ }^{87}$, and $\operatorname{cognition}^{88}$, which is another limitation. Despite these limitations, the observed pattern of higher working memory in patients with high 5-HI and low KYNA appears consistent with the directions of their mechanism of action ${ }^{19}$.

In summary, our findings suggest that there appears multiple potential mechanisms by which the tryptophan pathway is relevant to the cognitive performance in schizophrenia, including the indole branch whose relationship to schizophrenia has not been previously studied. Therefore, the finding that 5-HI and KYNA may have opposing effects on working memory among patients with schizophrenia is new and is consistent with preclinical evidences on their counteractive mechanisms. Our data may provide new insight into potential targets in the tryptophan pathway in our effort to develop novel therapeutic strategy for treating working memory and other cognitive impairment in schizophrenia.

\section{Acknowledgements \\ Support was received from the Capital' Funds for Health improvement and Research (CFH 2020-2-2132), the National Natural Science Foundation of China grants 81761128021 and 81771452 , the National Institutes of Health grants R01MH112180, and the Estonian Research Council-European Union Regional Developmental Fund Mobilitas Pluss Program number MOBTT77.}

\section{Author details}

'Peking University HuiLongGuan Clinical Medical School, Beijing Huilongguan Hospital, Beijing, China. ${ }^{2}$ Department of Pharmacy, Peking University First Hospital, Beijing, China. ${ }^{3}$ Maryland Psychiatric Research Center, Department of Psychiatry, University of Maryland School of Medicine, Baltimore, MD, USA. ${ }^{4}$ Faculty of Medicine, Department of Physiology, Institute of Biomedicine and Translational Medicine, University of Tartu, Tartu, Estonia

\section{Conflict of interest}

L.E.H. has received or plans to receive research funding or consulting fees on research projects from Mitsubishi, Your Energy Systems LLC, Neuralstem, Taisho, Heptares, Pfizer, Luye Pharma, Sound Pharma, Takeda, and Regeneron. None was involved in the design, analysis or outcomes of the study. All other authors declared that they have no conflicts of interest to this work and do not have any commercial or associative interest that represents a conflict of interest in connection with the work submitted.

\section{Publisher's note \\ Springer Nature remains neutral with regard to jurisdictional claims in published maps and institutional affiliations.}

Supplementary information The online version contains supplementary material available at https://doi.org/10.1038/s41398-021-01311-z.

Received: 25 September 2020 Revised: 19 February 2021 Accepted: 11 March 2021

Published online: 01 April 2021

\section{References}

1. Platten, M., Nollen, E. A. A., Rohrig, U. F., Fallarino, F. \& Opitz, C. A. Tryptophan metabolism as a common therapeutic target in cancer, neurodegeneration and beyond. Nat. Rev. Drug Discov. 18, 379-401 (2019).

2. Schwarcz, R., Bruno, J. P., Muchowski, P. J. \& Wu, H. Q. Kynurenines in the mammalian brain: when physiology meets pathology. Nat. Rev. Neurosci. 13, 465-477 (2012).

3. Alkondon, M. et al. Targeted deletion of the kynurenine aminotransferase ii gene reveals a critical role of endogenous kynurenic acid in the regulation of synaptic transmission via alpha7 nicotinic receptors in the hippocampus. J. Neurosci. 24, 4635-4648 (2004).

4. Hilmas, C. et al. The brain metabolite kynurenic acid inhibits alpha7 nicotinic receptor activity and increases non-alpha7 nicotinic receptor expression: physiopathological implications. J. Neurosci. 21, 7463-7473 (2001).

5. Levin, E. D. \& Simon, B. B. Nicotinic acetylcholine involvement in cognitive function in animals. Psychopharmacology 138, 217-230 (1998).

6. Felix, R. \& Levin, E. D. Nicotinic antagonist administration into the ventral hippocampus and spatial working memory in rats. Neuroscience $\mathbf{8 1}$, 1009-1017 (1997).

7. Martin, L. F. \& Freedman, R. Schizophrenia and the alpha7 nicotinic acetylcholine receptor. Int. Rev. Neurobiol. 78, 225-246 (2007).

8. Deutsch, S. I. et al. Therapeutic implications of a selective alpha7 nicotinic receptor abnormality in schizophrenia. Isr. J. psychiatry Relat. Sci. 42, 33-44 (2005).

9. Chess, A. C., Simoni, M. K., Alling, T. E. \& Bucci, D. J. Elevations of endogenous kynurenic acid produce spatial working memory deficits. Schizophr. Bull. 33, 797-804 (2007).

10. Schwarcz, R. et al. Increased cortical kynurenate content in schizophrenia. Biol. Psychiatry 50, 521-530 (2001).

11. Kindler, J. et al. Dysregulation of kynurenine metabolism is related to proinflammatory cytokines, attention, and prefrontal cortex volume in schizophrenia. Mol. Psychiatry https://doi.org/10.1038/s41380-41019-40401-41389 (2019).

12. Kessler, M., Terramani, T., Lynch, G. \& Baudry, M. A glycine site associated with $\mathrm{N}$-methyl-D-aspartic acid receptors: characterization and identification of a new class of antagonists. J. Neurochem. 52, 1319-1328 (1989).

13. Moghaddam, B. \& Javitt, D. From revolution to evolution: the glutamate hypothesis of schizophrenia and its implication for treatment. Neuropsychopharmacology 37, 4-15 (2012).

14. Javitt, D. C., Steinschneider, M., Schroeder, C. E. \& Arezzo, J. C. Role of cortical Nmethyl-D-aspartate receptors in auditory sensory memory and mismatch negativity generation: implications for schizophrenia. Proc. Natl Acad. Sci. USA 93, 11962-11967 (1996).

15. Driesen, N. R. et al. The impact of NMDA receptor blockade on human working memory-related prefrontal function and connectivity. Neuropsychopharmacology 38, 2613-2622 (2013).

16. Forsyth, J. K. et al. Effects of augmenting N-methyl-D-aspartate receptor signaling on qorking memory and experience-dependent plasticity in schizophrenia: an exploratory study using acute d-cycloserine. Schizophr. Bull. 43, 1123-1133 (2017).

17. Moroni, F. et al. Studies on the pharmacological properties of oxindole (2hydroxyindole) and 5-hydroxyindole: are they involved in hepatic encephalopathy? Adv. Exp. Med. Biol. 420, 57-73 (1997).

18. Zwart, R. et al. 5-Hydroxyindole potentiates human alpha 7 nicotinic receptormediated responses and enhances acetylcholine-induced glutamate release in cerebellar slices. Neuropharmacology 43, 374-384 (2002).

19. Grilli, M. et al. Modulation of the function of presynaptic alpha7 and nonalpha7 nicotinic receptors by the tryptophan metabolites, 5-hydroxyindole and kynurenate in mouse brain. Br. J. Pharmacol. 149, 724-732 (2006).

20. Schafer, J. A. \& Andreoli, T. E. Cellular constraints to diffusion. The effect of antidiuretic hormone on water flows in isolated mammalian collecting tubules. J. Clin. Investig. 51, 1264-1278 (1972).

21. Mannaioni, G., Carpenedo, R. \& Moroni, F. 5-hydroxyindole causes convulsions and increases transmitter release in the CA1 region of the rat hippocampus. $\mathrm{Br}$. J. Pharmacol. 138, 245-253 (2003).

22. Mok, M. H. \& Kew, J. N. Excitation of rat hippocampal interneurons via modulation of endogenous agonist activity at the alpha7 nicotinic ACh receptor. J. Physiol. 574, 699-710 (2006).

23. Beggiato, S. et al. Kynurenic acid, by targeting alpha7 nicotinic acetylcholine receptors, modulates extracellular GABA levels in the rat striatum in vivo. Eur. J. Neurosci. 37, 1470-1477 (2013). 
24. Lewis, D. A., Volk, D. W. \& Hashimoto, T. Selective alterations in prefrontal cortical GABA neurotransmission in schizophrenia: a novel target for the treatment of working memory dysfunction. Psychopharmacology 174, 143-150 (2004).

25. Lett, T. A., Voineskos, A. N., Kennedy, J. L., Levine, B. \& Daskalakis, Z. J. Treating working memory deficits in schizophrenia: a review of the neurobiology. Biol. Psychiatry 75, 361-370 (2014).

26. Tse, M. T., Piantadosi, P. T. \& Floresco, S. B. Prefrontal cortical gammaaminobutyric acid transmission and cognitive function: drawing links to schizophrenia from preclinical research. Biol. Psychiatry 77, 929-939 (2015).

27. Tavares, R. G. et al. Quinolinic acid stimulates synaptosomal glutamate release and inhibits glutamate uptake into astrocytes. Neurochem. Int. 40, 621-627 (2002).

28. Perkins, M. N. \& Stone, T. W. Quinolinic acid: regional variations in neuronal sensitivity. Brain Res. 259, 172-176 (1983).

29. Obrenovitch, T. P. Quinolinic acid accumulation during neuroinflammation. Does it imply excitotoxicity? Ann. N. Y. Acad. Sci. 939, 1-10 (2001)

30. Shear, D. A. et al. Chronic administration of quinolinic acid in the rat striatum causes spatial learning deficits in a radial arm water maze task. Exp. Neurol. 150, 305-311 (1998)

31. Block, F., Kunkel, M. \& Schwarz, M. Quinolinic acid lesion of the striatum induces impairment in spatial learning and motor performance in rats. Neurosci. Lett. 149, 126-128 (1993).

32. Latif-Hernandez, A. et al. Quinolinic acid injection in mouse medial prefrontal cortex affects reversal learning abilities, cortical connectivity and hippocampal synaptic plasticity. Sci. Rep. 6, 36489 (2016).

33. Chiappelli, J. et al. Salivary kynurenic acid response to psychological stress: inverse relationship to cortical glutamate in schizophrenia. Neuropsychopharmacology 43, 1706-1711 (2018).

34. Dominguez del Toro, E., Juiz, J. M., Peng, X., Lindstrom, J. \& Criado, M. Immunocytochemical localization of the alpha 7 subunit of the nicotinic acetylcholine receptor in the rat central nervous system. J. Comp. Neurol. 349, 325-342 (1994).

35. Carta, M., Srikumar, B. N., Gorlewicz, A., Rebola, N. \& Mulle, C. Activitydependent control of NMDA receptor subunit composition at hippocampal mossy fibre synapses. J. Physiol. 596, 703-716 (2018).

36. Andreasen, N., Pressler, M., Nopoulos, P., Miller, D. \& Ho, B. Antipsychotic dose equivalents and dose-years: a standardized method for comparing exposure to different drugs. Biol. Psychiatry 67, 255-262 (2010).

37. Gardner, D., Murphy, A., O'Donnell, H., Centorrino, F. \& Baldessarini, R. International consensus study of antipsychotic dosing. Am. J. Psychiatry 167, 686-693 (2010).

38. Shi, $\mathrm{C}$. et al. The MATRICS Consensus Cognitive Battery (MCCB): co-norming and standardization in China. Schizophrenia Res. 169, 109-115 (2015).

39. Nuechterlein, K. H. et al. The MATRICS Consensus Cognitive Battery, part 1: test selection, reliability, and validity. Am. J. Psychiatry 165, 203-213 (2008).

40. Kern, R. S. et al. The MATRICS Consensus Cognitive Battery, part 2: co-norming and standardization. Am. J. Psychiatry 165, 214-220 (2008).

41. Dickinson, D., Ramsey, M. E. \& Gold, J. M. Overlooking the obvious: a metaanalytic comparison of digit symbol coding tasks and other cognitive measures in schizophrenia. Arch. Gen. Psychiatry 64, 532-542 (2007).

42. Huang, J. et al. Hippocampus and cognitive domain deficits in treatmentresistant schizophrenia: a comparison with matched treatment-responsive

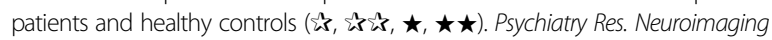
297, 111043 (2020).

43. Urbanska, E. M., Kocki, T., Saran, T., Kleinrok, Z. \& Turski, W. A. Impairment of brain kynurenic acid production by glutamate metabotropic receptor agonists. Neuroreport 8, 3501-3505 (1997).

44. Linderholm, K. R. et al. Increased levels of kynurenine and kynurenic acid in the CSF of patients with schizophrenia. Schizophr. Bull. 38, 426-432 (2012).

45. Sathyasaikumar, K. V. et al. Impaired kynurenine pathway metabolism in the prefrontal cortex of individuals with schizophrenia. Schizophr. Bull. 37, 1147-1156 (2011).

46. Fischl, B. et al. Whole brain segmentation: automated labeling of neuroanatomical structures in the human brain. Neuron 33, 341-355 (2002).

47. Desikan, R. S. et al. An automated labeling system for subdividing the human cerebral cortex on MRI scans into gyral based regions of interest. Neurolmage 31, 968-980 (2006).

48. Wong, T. Y. et al. An overlapping pattern of cerebral cortical thinning is associated with both positive symptoms and aggression in schizophrenia via the ENIGMA consortium. Psychol. Med. 50, 2034-2045 (2020).
49. King, L. J., Parke, D. V. \& Williams, R. T. The metabolism of [2-14C] indole in the rat. Biochem. J. 98, 266-277 (1966).

50. Bansal, T., Alaniz, R. C., Wood, T. K. \& Jayaraman, A. The bacterial signal indole increases epithelial-cell tight-junction resistance and attenuates indicators of inflammation. Proc. Natl Acad. Sci. USA 107, 228-233 (2010).

51. Karlin, D. A., Mastromarino, A. J., Jones, R. D., Stroehlein, J. R. \& Lorentz, O. Fecal skatole and indole and breath methane and hydrogen in patients with large bowel polyps or cancer. J. Cancer Res. Clin. Oncol. 109, 135-141 (1985).

52. Wikoff, W. R. et al. Metabolomics analysis reveals large effects of gut microflora on mammalian blood metabolites. Proc. Natl Acad. Sci. USA 106, 3698-3703 (2009).

53. Lee, J. H., Wood, T. K. \& Lee, J. Roles of indole as an interspecies and interkingdom signaling molecule. Trends Microbiol. 23, 707-718 (2015).

54. Carpenedo, R. \& Mannaioni, G. Moroni F. Oxindole, a sedative tryptophan metabolite, accumulates in blood and brain of rats with acute hepatic failure. J. Neurochem. 70, 1998-2003 (1998).

55. Greenberg, A. S. \& Coleman, M. Depressed 5-hydroxyindole levels associated with hyperactive and aggressive behavior. Relationship to drug response. Arch. Gen. Psychiatry 33, 331-336 (1976).

56. Szymona, K. et al. Correlations of kynurenic acid, 3-hydroxykynurenine, sIL-2R, IFN-alpha, and IL-4 with clinical symptoms during acute relapse of schizophrenia. Neurotox. Res. 32, 17-26 (2017).

57. Wurfel, B. E. et al. Serum kynurenic acid is reduced in affective psychosis. Transl. Psychiatry 7, e1115 (2017).

58. Chiappelli, J. et al. Influence of plasma cytokines on kynurenine and kynurenic acid in schizophrenia. Neuropsychopharmacology 43, 1675-1680 (2018).

59. Ravikumar, A., Deepadevi, K. V., Arun, P., Manojkumar, V. \& Kurup, P. A. Tryptophan and tyrosine catabolic pattern in neuropsychiatric disorders. Neurol. India 48, 231-238 (2000).

60. Barry, S., Clarke, G., Scully, P. \& Dinan, T. G. Kynurenine pathway in psychosis: evidence of increased tryptophan degradation. J. Psychopharmacol. 23, 287-294 (2009).

61. Plitman, E. et al. Kynurenic acid in schizophrenia: a systematic review and meta-analysis. Schizophr. Bull. 43, 764-777 (2017).

62. Fukui, S., Schwarcz, R., Rapoport, S. I., Takada, Y. \& Smith, Q. R. Blood-brain barrier transport of kynurenines: implications for brain synthesis and metabolism. J. Neurochem. 56, 2007-2017 (1991).

63. Pollak, T. A. et al. The blood-brain barrier in psychosis. Lancet Psychiatry $\mathbf{5}$ 79-92 (2018).

64. Kealy, J., Greene, C. \& Campbell, M. Blood-brain barrier regulation in psychiatric disorders. Neurosci. Lett. 726, 133664 (2020).

65. Owe-Young, R. et al. Kynurenine pathway metabolism in human blood-brainbarrier cells: implications for immune tolerance and neurotoxicity. J. Neurochem. 105, 1346-1357 (2008).

66. Chiappelli, J. et al. Stress-induced increase in kynurenic acid as a potential biomarker for patients with schizophrenia and distress intolerance. JAMA Psychiatry 71, 761-768 (2014).

67. Flores-Barrera, E. et al. Preferential disruption of prefrontal GABAergic function by nanomolar concentrations of the alpha7nACh negative modulator kynurenic acid. J. Neurosci. 37, 7921-7929 (2017).

68. Potter, M. C. et al. Reduction of endogenous kynurenic acid formation enhances extracellular glutamate, hippocampal plasticity, and cognitive behavior. Neuropsychopharmacology 35, 1734-1742 (2010).

69. Kozak, R. et al. Reduction of brain kynurenic acid improves cognitive function. J. Neurosci. 34, 10592-10602 (2014).

70. Stone, T. W. Neuropharmacology of quinolinic and kynurenic acids. Pharmacol. Rev. 45, 309-379 (1993).

71. Pellicciari, R. et al. Modulation of the kynurenine pathway in search for new neuroprotective agents. Synthesis and preliminary evaluation of (m-nitrobenzoyl)alanine, a potent inhibitor of kynurenine-3-hydroxylase. J. Med. Chem. 37, 647-655 (1994).

72. Marx, W. et al. The kynurenine pathway in major depressive disorder, bipolar disorder, and schizophrenia: a meta-analysis of 101 studies. Mol. Psychiatry 10.1038/s41380-020-00951-9 (2020).

73. Holmes, E. W., Russell, P. M., Kinzler, G. J. \& Bermes, E. W. Jr Inflammationassociated changes in the cellular availability of tryptophan and kynurenine in renal transplant recipients. Clin. Chim. Acta 227, 1-15 (1994).

74. Kegel, M. E. et al. Imbalanced kynurenine pathway in schizophrenia. Int. J. Tryptophan Res. 7, 15-22 (2014).

75. Lara, A. H., Kennerley, S. W. \& Wallis, J. D. Encoding of gustatory working memory by orbitofrontal neurons. J. Neurosci. 29, 765-774 (2009). 
76. LoPresti, M. L. et al. Working memory for social cues recruits orbitofrontal cortex and amygdala: a functional magnetic resonance imaging study of delayed matching to sample for emotional expressions. J. Neurosci. 28, 3718-3728 (2008).

77. Wallis, J. D., Anderson, K. C. \& Miller, E. K. Single neurons in prefrontal cortex encode abstract rules. Nature 411, 953-956 (2001)

78. Nogueira, R. et al. Lateral orbitofrontal cortex anticipates choices and integrates prior with current information. Nat. Commun. 8, 14823 (2017).

79. Cho, Y. T. et al. Effects of reward on spatial working memory in schizophrenia. J. Abnorm. Psychol. 127, 695-709 (2018).

80. Savitz, J. et al. Neuroprotective kynurenine metabolite indices are abnormally reduced and positively associated with hippocampal and amygdalar volume in bipolar disorder. Psychoneuroendocrinology 52, 200-211 (2015).

81. Young, K. D. et al. Kynurenine pathway metabolites are associated with hippocampal activity during autobiographical memory recall in patients with depression. Brain Behav. Immun. 56, 335-342 (2016).

82. Miller, B. J., Buckley, P., Seabolt, W., Mellor, A. \& Kirkpatrick, B. Meta-analysis of cytokine alterations in schizophrenia: clinical status and antipsychotic effects. Biol. Psychiatry 70, 663-671 (2011).
83. Noto, C. et al. Effects of risperidone on cytokine profile in drug-naïve firstepisode psychosis. Int. J. Neuropsychopharmacol. 18, pyu042 (2014).

84. Stojanovic, A. et al. Increased serum interleukin-6 levels in early stages of psychosis: associations with at-risk mental states and the severity of psychotic symptoms. Psychoneuroendocrinology 41, 23-32 (2014).

85. Schlittler, M. et al. Endurance exercise increases skeletal muscle kynurenine aminotransferases and plasma kynurenic acid in humans. Am. J. Physiol. Cell Physiol. 310, C836-840 (2016).

86. Lee, K. J. et al. High-fat diet and voluntary chronic aerobic exercise recover altered levels of aging-related tryptophan metabolites along the kynurenine pathway. Exp. Neurobiol. 26, 132-140 (2017).

87. Rogge, A. K., Röder, B., Zech, A. \& Hötting, K. Exercise-induced neuroplasticity: Balance training increases cortical thickness in visual and vestibular cortical regions. Neurolmage 179, 471-479 (2018).

88. Falkai, P., Malchow, B. \& Schmitt, A. Aerobic exercise and its effects on cognition in schizophrenia. Curr. Opin. Psychiatry 30, 171-175 (2017).

89. Stone, T. W., Forrest, C. M. \& Darlington, L. G. Kynurenine pathway inhibition as a therapeutic strategy for neuroprotection. FEBS J. 279, 1386-1397 (2012).

90. Myint, A. M. Kynurenines: from the perspective of major psychiatric disorders. FEBS J. 279, 1375-1385 (2012). 